\title{
Epistémologie et didactique de la physique : le constructivisme en question
}

\section{Manuel Bächtold}

\section{(2) OpenEdition \\ Journals}

Édition électronique

URL : http://journals.openedition.org/trema/2815

DOI : $10.4000 /$ trema.2815

ISSN : 2107-0997

Éditeur

Faculté d'Éducation de l'université de Montpellier

Édition imprimée

Date de publication : 1 décembre 2012

Pagination : 1-5

ISBN : 1167-315X

ISSN : $1167-315 X$

Référence électronique

Manuel Bächtold, «Epistémologie et didactique de la physique : le constructivisme en question », Tréma [En ligne], 38 | 2012, mis en ligne le 01 décembre 2012, consulté le 22 septembre 2020. URL http://journals.openedition.org/trema/2815 ; DOI : https://doi.org/10.4000/trema.2815

Ce document a été généré automatiquement le 22 septembre 2020.

Trema 


\title{
Epistémologie et didactique de la physique : le constructivisme en question
}

\author{
Manuel Bächtold
}

1 Ce numéro de Tréma vise à interroger la suprématie du constructivisme en didactique des sciences et, en particulier, en didactique de la physique. Ce courant de pensée repose sur une certaine conception de l'apprentissage. Le noyau de cette conception, qui se retrouve dans les multiples variantes du constructivisme, peut être formulé ainsi: pour apprendre de nouvelles connaissances, les élèves doivent s'impliquer activement sur plan intellectuel en construisant par eux-mêmes ces connaissances.

2 Rappelons que le constructivisme a émergé à la fin des années 1970 et au début des années 1980, alors même que la didactique des sciences se constituait comme nouveau domaine de recherche (Solomon, 1994). Depuis les années 1980, le constructivisme a été adopté par un nombre très élevé et toujours croissant d'auteurs dans ce domaine (Philipps, 1995, Matthews, 1998). Omniprésent dans la littérature en didactique des sciences, le constructivisme en est venu à exercer une forte influence sur les acteurs du système éducatif (enseignants, formateurs, inspecteurs...). Cette influence s'est notamment traduite, dans les programmes officiels de nombreux pays, par la préconisation de l'enseignement des sciences basé sur l'investigation (désigné en anglais par les expressions Inquiry-based Science Education ou Inquiry-based Science Teaching). Soulignons toutefois que l'investigation n'est que l'un des moyens envisagés pour favoriser l'implication des élèves dans la construction des connaissances à enseigner. Plus fondamentalement, les différents promoteurs du constructivisme préconisent un enseignement des sciences qui prend pour point de départ les conceptions des élèves (Baviskar et al., 2009) : l'objectif est que les élèves explicitent leurs conceptions, prennent conscience de leur insuffisance et s'engagent dans une démarche permettant la construction de conceptions qui soient satisfaisantes du point de vue des élèves et qui idéalement correspondent aux connaissances visées par l'enseignant. 
3 L'idée de la construction des connaissances par les élèves est très fréquemment formulée dans la littérature de recherche et dans les textes officiels. Toutefois, elle n'est que rarement éclairée et développée. En quel sens les élèves sont-ils supposés « construire » les connaissances?

4 Telle est la question que le premier article de ce numéro de Tréma, intitulé «Les fondements constructivistes de l'enseignement des sciences basé sur l'investigation ", entend explorer. À travers une synthèse de la littérature en didactique des sciences depuis la fin des années 1970, nous (Manuel Bächtold) proposons dans cet article de clarifier quelles sont les sources d'influence théorique du constructivisme en didactique des sciences. Ces sources d'influence proviennent à la fois du domaine de la psychologie du développement cognitif et de celui de l'épistémologie. Nous cherchons à montrer que cette dualité au niveau des sources d'influence conduit à deux points de vue sur l'apprentissage des sciences et, corrélativement, à deux façons d'entendre le terme "construction ». En effet, à la lumière de la psychologie du développement cognitif, le processus de construction mené par les élèves en classe est conçu comme une extension du processus cognitif spontané et inconscient prenant place avant et en dehors de la classe, plus précisément un processus de construction ou de réorganisation des structures cognitives. À la lumière de l'épistémologie, ce processus de construction mené par les élèves en classe est conçu par analogie avec l'activité de recherche des scientifiques lorsqu'ils construisent de nouveaux modèles ou de nouvelles théories.

5 Si, depuis les années 1980 , le constructivisme a exercé une très forte influence en didactique des sciences, au point de devenir selon certains le nouveau "paradigme " (Tobin, 1993, p. ix), cela n'a pas été sans susciter certaines réactions hostiles. Le titre d'un article de Jenkins (2000), reprenant deux citations d'auteurs différents, illustre la réception ambivalente $\mathrm{du}$ constructivisme: "Constructivism in school science education : powerful model or the most dangerous intellectual tendency?». Plusieurs critiques ont été formulées à l'encontre de ce courant de pensée, et ce principalement dans les années 1990 (Millar, 1989, Osborne, 1996, Nola, 1997, Matthews, 1997, Kelly, 1997, Martinez-Delgado, 2002, etc.). Ces critiques n'ont pas manqué de susciter des répliques (Duit, 1995, Staver, 1998, Quale, 2007, etc.). À noter que ce débat s'est focalisé essentiellement sur une forme particulière de constructivisme, à savoir le constructivisme « radical» de von Glasersfeld (1995). Les articles de Michael Matthews et Alberto Martinez Delgado figurant dans ce numéro de Tréma réactualisent et prolongent ces critiques.

6 Dans son article "Philosophical and pedagogical problems with constructivism in science education ", Michael Matthews identifie le constructivisme à une idéologie et à une épistémologie à la fois relativiste (subjectiviste), sceptique et idéaliste. Il reproche à cette théorie d'opérer une confusion entre la psychologie et l'épistémologie, et en particulier, entre l'apprentissage (de la connaissance) et la connaissance (elle-même). Mais ce qui constitue peut-être la principale objection de Michael Matthews porte sur la thèse anti-réaliste suivante qu'il associe au constructivisme : si un concept est une construction humaine, il ne fait pas référence à quelque chose qui existe. Cette thèse est, d'après l'auteur, un « dogme » sans fondement et conduit à l'absurde (le référent de "je», qui est une construction humaine, n'existerait pas non plus). Les différentes critiques qu'il adresse au constructivisme le motivent à exiger, dans sa conclusion, un fondement philosophique plus solide pour la didactique des sciences. 
7 Alberto Martinez Delgado, dans son article intitulé «Le constructivisme radical et Socrate: aspects pédagogiques et idéologiques ", opère d'abord un rapprochement original entre le constructivisme radical de von Glasersfeld et la théorie de Socrate et Platon, au niveau non seulement de leur conception idéaliste de la connaissance, mais aussi des méthodes pédagogiques consistant à faire émerger chez les élèves des connaissances préexistantes. À l'occasion de cette analyse comparative, l'auteur propose une critique des deux théories : leur conception de la connaissance occulte la réalité extérieure au sujet et, par conséquent, elles se révèlent incapables d'expliquer que les élèves puissent retrouver les connaissances préexistantes visées dans l'enseignement. Il développe ensuite sa critique du constructivisme radical en se centrant sur plusieurs thèses et préconisations pédagogiques de cette théorie, en particulier sur son rejet de l'enseignement transmissif. Dans la dernière partie de son article, Alberto Martinez Delgado met en lumière la dimension idéologique (postmoderne) du constructivisme radical en ce qu'il fait de tout individu le seul responsable de son propre vécu et dénonce l'utilisation qui est faite actuellement de cette idéologie par la classe financière dominante.

8 Dans l'article suivant, "The supremacy of the constructivist appraoch in the field of physics education: myths and real challenges ", Amparo Vilches et Daniel Gil-Pérez semblent prendre le contre-pieds du premier paragraphe de ce cette présentation ainsi que des auteurs des deux articles précédents, en soutenant que le constructivisme n'est en réalité nullement dominant et que sa prétendue suprématie n'est qu'un «mythe ». En effet, d'après ces auteurs, le constructivisme en didactique des sciences, qui ne se réduit pas au constructivisme radical de von Glasersfeld, constitue certes la perspective dominante dans le domaine de la recherche, mais en pratique (c'est-à-dire dans les classes) n'est guère mis en œuvre, encore éclipsé par le modèle de la transmission/ réception. C'est ce dernier, considéré comme "dominant», que les auteurs se proposent donc de soumettre à la critique. Celui-ci, soutiennent-ils, véhicule une image de la science à la fois pauvre et déformée : la science serait socialement neutre, les connaissances seraient produites par des savants isolés et de façon linéaire et cumulative, l'observation et l'expérimentation seraient neutres du point de vue théorique, ou encore la méthode scientifique se réduirait à une séquence d'étapes appliquées mécaniquement. Afin que les élèves comprennent la manière dont les connaissances scientifiques sont réellement construites et accèdent à la signification de ces connaissances, Amparo Vilches et Daniel Gil-Pérez plaident pour un enseignement des sciences rénové qui rende mieux compte de la nature de la science, en particulier de son caractère ouvert et créatif (invention d'hypothèses provisoires). Ils proposent ainsi plusieurs pistes, telles que inviter les élèves à discuter de l'intérêt possible des situations étudiées ou les impliquer dans l'invention de concepts et d'hypothèses.

9 Dans le dernier article de ce numéro, « Réflexions sur l'articulation entre didactique et épistémologie dans le domaine des grandeurs et mesures dans l'enseignement primaire et secondaire ", Valérie Munier semble rejoindre les auteurs de l'article précédent sur l'intérêt d'initier les élèves à l'épistémologie dans l'enseignement des sciences. Son analyse porte, non pas sur le constructivisme ou la didactique de la physique en général, mais sur deux concepts spécifiques qui se retrouvent dans l'enseignement de la physique tout au long de la scolarité : ceux de grandeur et de mesure. L'auteur précise la signification de ces deux concepts, qui sont intimement liés, et souligne qu'ils peuvent être compris schématiquement selon deux points de vue épistémologiques 
opposés : un point de vue réaliste selon lequel les grandeurs se réfèrent à une réalité dont l'existence est indépendante de toute mesure (ou " mesurage ») et un point de vue opérationaliste selon lequel les grandeurs n'ont pas de signification indépendamment de leur mesure. À la lumière de cette clarification, elle examine les programmes scolaires, de l'école au lycée, et discute les points de vue épistémologiques tacitement adoptés. Elle offre ensuite une synthèse des études récentes dans le domaine de la didactique de la physique à propos de ces deux concepts. Celles-ci pointent les difficultés récurrentes des élèves à saisir la signification des différentes grandeurs enseignées et en particulier leurs liens avec les phénomènes et les instruments de mesure. Valérie Munier estime que pour palier ces difficultés, il est nécessaire d'introduire des savoirs d'ordre épistémologique et des éléments de métrologie dans l'enseignement pour permettre aux élèves de donner du sens aux concepts de grandeur et de mesure.

Les différents auteurs de ce numéro de Tréma semblent nous éloigner de la thèse antiréaliste, centrale dans le constructivisme radical de von Glasersfeld, selon laquelle les connaissances scientifiques ne fournissent pas «des représentations d'une réalité indépendante " (von Glasersfeld, 2005, p. 3). En fin de compte, le débat entre réalisme et anti-réalisme ne présente peut-être que peu d'intérêt pour la recherche en didactique de la physique. Quel est donc l'apport de l'épistémologie dans ce domaine? Les articles de ce numéro suggèrent l'idée suivante: l'éclairage apporté par l'épistémologie sur la manière dont les connaissances sont construites par les scientifiques peut s'avérer très fécond pour la compréhension de ces connaissances, de sorte que tant enseignants qu'élèves gagneraient à être initiés à la perspective réflexive de l'épistémologie. Ce numéro de Tréma entend ainsi alimenter la discussion sur les liens entre l'épistémologie et la didactique de la physique.

\section{BIBLIOGRAPHIE}

Baviskar, S., Hartle, T. et Whitney, T., Essential criteria to characterize constructivist teaching : derived from a review of the literature and applied to five constructivist-teaching method articles, International Journal of Science Education, 31 (4), 2009, p. 541-550.

Duit, R., The constructivist view : a fashionable and fruitful paradigm for science education. Dans L. Steffe et J. Gale (eds.), Constructivism in education. Hillsdale (New Jersey) : Erlbaum. 1995, p. 271-285.

Jenkins, E., Constructivism in school science education : powerful model or the most dangerous intellectual tendency? Science et Education, 9, 2000, p. 599-610.

Kelly, G., Research traditions in comparative context : a philosophical challenge to radical constructivism, Science Education, 81, 1997, p. 355-375.

Martínez-Delgado, A., Radical constructivism : between realism and solipsism, Science Education, 86,2002 , p. $840-855$. 
Matthews, M. R., Introductory comments on philosophy and constructivism in science education, Science et Education, 6 (1-2), 1997, p. 5-14.

Matthews, M., Preface. Dans M. Matthews (ed.), Constructivism in science education : a philosophical examination (pp. ix-xii). Dordrecht, Boston and London : Kluwer. 1998.

Millar, R., Constructive criticisms, International Journal of Science Education, 11 (5), 1989, p. 587-596. Nola, R., Constructivism in science and in science education : a philosophical critique, Science et Education, 6 (1-2), 1997, p. 55-83.

Osborne, J. F., Beyond constructivism, Science Education, 80 (1), 1996, p. 53-82.

Phillips, D. The good, the bad and the ugly : the many faces of constructivism, Educational Researcher, 24 (7), 1995, p. 5-12.

Quale, A., Radical constructivism, and the sin of relativism, Science et Education, 16, 2007, p. 231-266.

Solomon, J., The rise and fall of constructivism, Studies in Science Education, 23, 1994, p. 1-19.

Staver, J., Constructivism : sound theory for explicating the practice of science and science teaching, Journal of Research in Science Teaching, 35 (5), 1998, p. 501-520.

Tobin, K., (ed.) The practice of constructivism in science and mathematics education. Washington DC : AAA Press. 1993.

Von Glasersfeld, E., Radical constructivism : a way of knowing and learning. Londres, Washington : The Falmer Press. 1995.

Von Glasersfeld, E., Introduction : aspects of constructivism. Dans C. T. Fosnot (ed.), Constructivism : theory, perspectives, and practice. New York et Londres : Teachers College Press. 2005, p. 3-7.

\section{AUTEUR}

\section{MANUEL BÄCHTOLD}

LIRDEF (EA 3749), IUFM de l'académie de Montpellier - UM2 et Université Montpellier 3 